\title{
WEIGHTED POINCARÉ-TYPE ESTIMATES FOR CONJUGATE $A$-HARMONIC TENSORS
}

YUMING XING

Received 14 August 2004

We prove Poincaré-type estimates involving the Hodge codifferential operator and Green's operator acting on conjugate $A$-harmonic tensors.

\section{Preliminary}

In a survey paper [1], Agarwal and Ding summarized the advances achieved in the study of $A$-harmonic equations. Some recent results about $A$-harmonic equations can also be found in $[2,3,5,6]$. The purpose of this note is to establish some estimates about Green's operator and the Hodge codifferential operator $d^{*}$, which will enrich the existing literature in the field of $A$-harmonic equations.

Let $\Omega$ be a connected open subset of $\mathbb{R}^{n}, n \geq 2, B$ a ball in $\mathbb{R}^{n}$ and $\rho B$ denote the ball with the same center as $B$ and with $\operatorname{diam}(\rho B)=\rho \operatorname{diam}(B)$. The $n$-dimensional Lebesgue measure of a set $E \subseteq \mathbb{R}^{n}$ is denoted by $|E|$. We call $w$ a weight if $w \in L_{\text {loc }}^{1}\left(\mathbb{R}^{n}\right)$ and $w>0$ a.e. For $0<p<\infty$ and a weight $w(x)$, we denote the weighted $L^{p}$-norm of a measurable function $f$ over $E$ by $\|f\|_{p, E, w^{\alpha}}=\left(\int_{E}|f(x)|^{p} w^{\alpha} d x\right)^{1 / p}$, where $\alpha$ is a real number. Let $\Lambda^{l}=\Lambda^{l}\left(\mathbb{R}^{n}\right)$ be the linear space of all $l$-forms $\omega(x)=\sum_{I} \omega_{I}(x) d x_{I}=\sum \omega_{i_{1} i_{2} \cdots i_{l}}(x) d x_{i_{1}} \wedge$ $d x_{i_{2}} \wedge \cdots \wedge d x_{i_{l}}, l=0,1, \ldots, n$. Assume that $D^{\prime}\left(\Omega, \Lambda^{l}\right)$ is the space of all differential $l$ forms and $L^{p}\left(\Omega, \Lambda^{l}\right)$ is the space of all $L^{p}$-integrable $l$-forms, which is a Banach space with norm $\|\omega\|_{p, \Omega}=\left(\int_{\Omega}|\omega(x)|^{p} d x\right)^{1 / p}=\left(\int_{\Omega}\left(\sum_{I}\left|\omega_{I}(x)\right|^{2}\right)^{p / 2} d x\right)^{1 / p}$. We denote the exterior derivative by $d: D^{\prime}\left(\Omega, \Lambda^{l}\right) \rightarrow D^{\prime}\left(\Omega, \Lambda^{l+1}\right)$ for $l=0,1, \ldots, n-1$. Its formal adjoint operator $d^{*}: D^{\prime}\left(\Omega, \Lambda^{l+1}\right) \rightarrow D^{\prime}\left(\Omega, \Lambda^{l}\right)$ is given by $d^{*}=(-1)^{n l+1} * d *$ on $D^{\prime}\left(\Omega, \Lambda^{l+1}\right), l=$ $0,1, \ldots, n-1$, where $*$ is the Hodge star operator. We call $u$ and $v$ a pair of conjugate $A$-harmonic tensor in $\Omega$ if $u$ and $v$ satisfy the conjugate $A$-harmonic equation

$$
A(x, d u)=d^{*} v
$$

in $\Omega$, where $A: \Omega \times \Lambda^{l}\left(\mathbb{R}^{n}\right) \rightarrow \Lambda^{l}\left(\mathbb{R}^{n}\right)$ satisfies conditions: $|A(x, \xi)| \leq a|\xi|^{p-1}$ and $\langle A(x, \xi), \xi\rangle \geq|\xi|^{p}$ for almost every $x \in \Omega$ and all $\xi \in \Lambda^{l}\left(\mathbb{R}^{n}\right)$. Here $a>0$ is a constant. In this paper, we always assume that $p$ is the fixed exponent associated with $(1.1), 1<p<\infty$ and $p^{-1}+q^{-1}=1$. 
The following weak reverse Hölder inequality about $d^{*} v$ appears in [3].

Lemma 1.1. Let $u$ and $v$ be a pair of solutions of (1.1) in $\Omega, \sigma>1$ and $0<s, t<\infty$. Then there exists a constant $C$, independent of $v$, such that $\left\|d^{*} v\right\|_{s, B} \leq C|B|^{(t-s) / s t}\left\|d^{*} v\right\|_{t, \sigma B}$ for all balls $B$ with $\sigma B \subset \Omega$.

Setting the differential form $u=d^{*} v$ in [2, Corollary 2.6], we obtain the following Poincaré-type inequality for Green's operator.

$$
\left\|G\left(d^{*} v\right)-\left(G\left(d^{*} v\right)\right)_{B}\right\|_{p, B} \leq C\left\|d^{*} v\right\|_{p, B} .
$$

Definition 1.2. A weight $w(x)$ is called an $A_{r}$-weight for some $r>1$ on a subset $E \subset \mathbb{R}^{n}$, write $w \in A_{r}(E)$, if $w(x)>0$ a.e., and

$$
\sup _{B}\left(\frac{1}{|B|} \int_{B} w d x\right)\left(\frac{1}{|B|} \int_{B}\left(\frac{1}{w}\right)^{1 /(r-1)} d x\right)^{(r-1)}<\infty
$$

for any ball $B \subset E$.

We also need the following well-known reverse Hölder inequality for $A_{r}$-weights.

Lemma 1.3. If $w \in A_{r}$, then there exist constants $\beta>1$ and $C$, independent of $w$, such that $\|w\|_{\beta, B} \leq C|B|^{(1-\beta) / \beta}\|w\|_{1, B}$ for all balls $B \subset \mathbb{R}^{n}$.

The following generalized Hölder inequality will be used repeatedly in this paper.

Lemma 1.4. Let $0<\alpha<\infty, 0<\beta<\infty$, and $s^{-1}=\alpha^{-1}+\beta^{-1}$. If $f$ and $g$ are measurable functions on $\mathbb{R}^{n}$, then $\|f g\|_{s, E} \leq\|f\|_{\alpha, E} \cdot\|g\|_{\beta, E}$ for any $E \subset \mathbb{R}^{n}$.

The following lemma appears in [6].

Lemma 1.5. Let $u$ and $v$ be a pair of solutions of (1.1) in a domain $\Omega$. Then, there exists a constant $C$, independent of $u$ and $v$, such that

$$
\|d u\|_{p, D, w^{\alpha}}^{p} \leq\left\|d^{*} v\right\|_{q, D, w^{\alpha}}^{q} \leq C\|d u\|_{p, D, w^{\alpha}}^{p}
$$

for any subset $D \subset \Omega$. Here $w$ is any weight and $\alpha>0$ is any constant.

\section{Main results and proofs}

Now, we prove the following $A_{r}$-weighted Poincaré-type inequality for Green's operator $G$ acting on solutions of (1.1).

THEOREm 2.1. Let $u$ and $v$ be a pair of solutions of (1.1) in $\Omega$, and assume that $\omega \in A_{r}(\Omega)$ for some $r>1, \sigma>1,0<\alpha \leq 1$, and $1+\alpha(r-1)<q<\infty$. Then, there exists a constant $C$, independent of $u$ and $v$, such that

$$
\left\|G\left(d^{*} v\right)-\left(G\left(d^{*} v\right)\right)_{B}\right\|_{q, B, w^{\alpha}}^{q} \leq C\|d u\|_{p, \sigma B, w^{\alpha}}^{p}
$$

for all balls $B$ with $\sigma B \subset \Omega$. 
Proof. First, we assume that $0<\alpha<1$. Let $s=q /(1-\alpha)$. Using Hölder inequality we get

$$
\begin{aligned}
\left(\int_{B} \mid\right. & \left.G\left(d^{*} v\right)-\left.\left(G\left(d^{*} v\right)\right)_{B}\right|^{q} w^{\alpha} d x\right)^{1 / q} \\
\leq & \left(\int_{B}\left(\left|G\left(d^{*} v\right)-\left(G\left(d^{*} v\right)\right)_{B}\right| w^{\alpha / q}\right)^{q} d x\right)^{1 / q} \\
\leq & \left(\int_{B}\left|G\left(d^{*} v\right)-\left(G\left(d^{*} v\right)\right)_{B}\right|^{s} d x\right)^{1 / s}\left(\int_{B} w^{\alpha s /(s-q)} d x\right)^{(s-q) / q s} \\
& =\left\|G\left(d^{*} v\right)-\left(G\left(d^{*} v\right)\right)_{B}\right\|_{s, B}\left(\int_{B} w d x\right)^{\alpha / q} .
\end{aligned}
$$

Select $t=q /(\alpha(r-1)+1)$, then $t<q$. Using Lemma 1.1 and (1.2), we find that

$$
\left\|G\left(d^{*} v\right)-\left(G\left(d^{*} v\right)\right)_{B}\right\|_{s, B} \leq C_{1}\left\|d^{*} v\right\|_{s, B} \leq C_{2}|B|^{(t-s) / t s}\left\|d^{*} v\right\|_{t, \sigma B}
$$

for all balls $B$ with $\sigma B \subset \Omega$. Since $1 / t=1 / q+(q-t) / q t$, by Hölder inequality again, we have

$$
\begin{aligned}
\left\|d^{*} v\right\|_{t, \sigma B} & =\left(\int_{\sigma B}\left(\left|d^{*} v\right| w^{\alpha / q} w^{-\alpha / q}\right)^{t} d x\right)^{1 / t} \\
& \leq\left(\int_{\sigma B}\left|d^{*} v\right|^{q} w^{\alpha} d x\right)^{1 / q}\left(\int_{\sigma B}\left(\frac{1}{w}\right)^{\alpha t /(q-t)} d x\right)^{(q-t) / q t} \\
& =\left(\int_{\sigma B}\left|d^{*} v\right|^{q} w^{\alpha} d x\right)^{1 / q}\left(\int_{\sigma B}\left(\frac{1}{w}\right)^{1 /(r-1)} d x\right)^{\alpha(r-1) / q} .
\end{aligned}
$$

Combining (2.2), (2.3), and (2.4) yields

$$
\begin{aligned}
& \left(\int_{B}\left|G\left(d^{*}\right)-\left(G\left(d^{*} v\right)\right)_{B}\right|^{q} w^{\alpha} d x\right)^{1 / q} \\
& \quad \leq C_{2}|B|^{(t-s) / t s}\left(\int_{B} w d x\right)^{\alpha / q}\left(\int_{\sigma B}\left(\frac{1}{w}\right)^{1 /(r-1)} d x\right)^{\alpha(r-1) / q}\left(\int_{\sigma B}\left|d^{*} v\right|^{q} w^{\alpha} d x\right)^{1 / q} .
\end{aligned}
$$

Noting that $w \in A_{r}$, we have

$$
\begin{aligned}
& \left(\int_{B} w d x\right)^{\alpha / q}\left(\int_{\sigma B}\left(\frac{1}{w}\right)^{1 /(r-1)} d x\right)^{\alpha(r-1) / q} \\
& \quad \leq\left(|\sigma B|^{r}\left(\frac{1}{|\sigma B|} \int_{\sigma B} w d x\right)\left(\frac{1}{|\sigma B|} \int_{\sigma B}\left(\frac{1}{w}\right)^{1 /(r-1)} d x\right)^{(r-1)}\right)^{\alpha / q} \leq C_{3}|B|^{\alpha r / q} .
\end{aligned}
$$

Substituting (2.6) into (2.5) with $(t-s) / t s+\alpha r / q=0$, it follows that

$$
\left(\int_{B}\left|G\left(d^{*} v\right)-\left(G\left(d^{*} v\right)\right)_{B}\right|^{q} w^{\alpha} d x\right)^{1 / q} \leq C_{4}\left(\int_{\sigma B}\left|d^{*} v\right|^{q} w^{\alpha} d x\right)^{1 / q} .
$$


Applying Lemma 1.5 and (2.7), we conclude that

$$
\left\|G\left(d^{*} v\right)-\left(G\left(d^{*} v\right)\right)_{B}\right\|_{q, B, w^{\alpha}}^{q} \leq C_{5}\left\|d^{*} v\right\|_{q, \sigma B, w^{\alpha}}^{q} \leq C_{6}\|d u\|_{p, \sigma B, w^{\alpha}}^{p} .
$$

We have proved that $(2.1)$ is true if $0<\alpha<1$.

Next, we show that (2.1) is also true for $\alpha=1$. By Lemma 1.3, there exist constants $\beta>1$ and $C_{7}>0$, such that

$$
\|w\|_{\beta, B} \leq C_{7}|B|^{(1-\beta) / \beta}\|w\|_{1, B}
$$

for any ball $B \subset \mathbb{R}^{n}$. Choose $s=q \beta /(\beta-1)$, then $1<q<s$ and $\beta=s /(s-q)$. Since $1 / q=$ $1 / s+(s-q) / q s$, using Lemma 1.4 and (2.9), we obtain

$$
\begin{aligned}
\left(\int_{B} \mid\right. & \left.G\left(d^{*} v\right)-\left.\left(G\left(d^{*} v\right)\right)_{B}\right|^{q} w d x\right)^{1 / q} \\
\leq & \left(\int_{B}\left|G\left(d^{*} v\right)-\left(G\left(d^{*} v\right)\right)_{B}\right|^{s} d x\right)^{1 / s}\left(\int_{B}\left(w^{1 / q}\right)^{q s /(s-q)} d x\right)^{(s-q) / s q} \\
& =\left\|G\left(d^{*} v\right)-\left(G\left(d^{*} v\right)\right)_{B}\right\|_{s, B} \cdot\|w\|_{\beta, B}^{1 / q} \\
& \leq C_{8}\left\|G\left(d^{*} v\right)-\left(G\left(d^{*} v\right)\right)_{B}\right\|_{s, B} \cdot|B|^{(1-\beta) / \beta q}\|w\|_{1, B}^{1 / q} .
\end{aligned}
$$

Now, choose $t=q / r$, then $t<q$. From Lemma 1.1 and (1.2), we have

$$
\left\|G\left(d^{*} v\right)-\left(G\left(d^{*} v\right)\right)_{B}\right\|_{s, B} \leq C_{9}\left\|d^{*} v\right\|_{s, B} \leq C_{10}|B|^{(t-s) / s t}\left\|d^{*} v\right\|_{t, \sigma B} .
$$

Using Hölder inequality again, we find that

$$
\begin{aligned}
\left\|d^{*} v\right\|_{t, \sigma B} & =\left(\int_{\sigma B}\left(\left|d^{*} v\right| w^{1 / q} w^{-1 / q}\right)^{t} d x\right)^{1 / t} \\
& \leq\left(\int_{\sigma B}\left|d^{*} v\right|^{q} w d x\right)^{1 / q}\left(\int_{\sigma B}\left(\frac{1}{w}\right)^{t /(q-t)} d x\right)^{(q-t) / q t} \\
& =\left(\int_{\sigma B}\left|d^{*} v\right|^{q} w d x\right)^{1 / q}\left(\int_{\sigma B}\left(\frac{1}{w}\right)^{1 /(r-1)} d x\right)^{(r-1) / q} .
\end{aligned}
$$

Combining (2.11) and (2.12) yields

$$
\begin{aligned}
& \left\|G\left(d^{*} v\right)-\left(G\left(d^{*} v\right)\right)_{B}\right\|_{s, B} \\
& \quad \leq C_{11}|B|^{(t-s) / s t}\left(\int_{\sigma B}\left|d^{*} v\right|^{q} w d x\right)^{1 / q}\left(\int_{\sigma B}\left(\frac{1}{w}\right)^{1 /(r-1)} d x\right)^{(r-1) / q} .
\end{aligned}
$$

Since $w \in A_{r}$, we obtain

$$
\left(\int_{B} w d x\right)^{1 / q}\left(\int_{\sigma B}\left(\frac{1}{w}\right)^{1 /(r-1)} d x\right)^{(r-1) / q} \leq C_{12}|B|^{r / q}
$$


Substituting (2.13) into (2.10) and using (2.14), we find that

$$
\begin{aligned}
& \left\|G\left(d^{*} v\right)-\left(G\left(d^{*} v\right)\right)_{B}\right\|_{q, B, w} \\
& \quad \leq C_{13}|B|^{(1-\beta) / \beta q}|B|^{(t-s) / s t}|| d^{*} v\left\|_{q, \sigma B, w}\right\| w \|_{1, B}^{1 / q}\left(\int_{\sigma B}\left(\frac{1}{w}\right)^{1 /(r-1)} d x\right)^{(r-1) / q} \\
& \quad \leq C_{14}|B|^{(1-\beta) / \beta q}|B|^{(t-s) / s t}|B|^{r / q}\left\|d^{*} v\right\|_{q, \sigma B, w} \leq C_{15}\left\|d^{*} v\right\|_{q, \sigma B, w} .
\end{aligned}
$$

Combining Lemma 1.5 and (2.15), we conclude that

$$
\left\|G\left(d^{*} v\right)-\left(G\left(d^{*} v\right)\right)_{B}\right\|_{q, B, w}^{q} \leq C_{16}\left\|d^{*} v\right\|_{q, \sigma B, w}^{q} \leq C_{17}\|d u\|_{p, \sigma B, w}^{p} .
$$

This ends the proof of Theorem 2.1.

For any weight $w$, we define the weighted norm of $\omega \in W^{1, p}\left(\Omega, \Lambda^{l}, w^{\alpha}\right)$ in $\Omega$ by

$$
\|\omega\|_{W^{1, p}(\Omega), w^{\alpha}}=\operatorname{diam}(\Omega)^{-1}\|\omega\|_{p, \Omega, w^{\alpha}}+\|\nabla \omega\|_{p, \Omega, w^{\alpha}}, \quad 0<p<\infty .
$$

Now we can give the following Sobolev norm estimates for Green operator in terms of Hodge codifferential operator.

THEOREM 2.2. Let $u$ and $v$ be a pair of solutions of (1.1) in $\Omega$, and assume that $\omega \in A_{r}(\Omega)$ for some $r>1, \sigma>1,0<\alpha \leq 1$, and $r<p<\infty$. Then, there exists a constant $C$, independent of $u$ and $v$, such that

$$
\left\|G(u)-(G(u))_{B}\right\|_{W^{1, p}(B), w^{\alpha}}^{p} \leq C\left\|d^{*} v\right\|_{q, \sigma B, w^{\alpha}}^{q}
$$

for all balls $B$ with $\sigma B \subset \Omega$. Here $\alpha$ is any constant with $0<\alpha \leq 1$.

Proof. We know that Green's operator commutes with $d$ in [4], that is, for any smooth differential form $u$, we have $d G(u)=G d(u)$. Since $|\nabla \omega|=|d \omega|$ for any differential form $\omega$, we have $\|\nabla G(u)\|_{p, B}=\|d G(u)\|_{p, B}=\|G(d u)\|_{p, B} \leq C_{1}\|d u\|_{p, B}$ from [2, Lemma 2.1]. Using the same method as we did above, we can also have the following $A_{r}$-weighted inequalities

$$
\begin{gathered}
\left\|G(u)-(G(u))_{B}\right\|_{p, B, w^{\alpha}} \leq C_{2} \operatorname{diam}(B)\|d u\|_{p, \sigma B, w^{\alpha}}, \\
\left\|\nabla\left(G(u)-(G(u))_{B}\right)\right\|_{p, B, w^{\alpha}} \leq C_{3}\|d u\|_{p, \sigma B, w^{\alpha}} .
\end{gathered}
$$

Combining (2.17) and (2.19), it follows that

$$
\begin{aligned}
\| G(u) & -(G(u))_{B} \|_{W^{1, p}(B), w^{\alpha}} \\
& =\operatorname{diam}(B)^{-1}\left\|G(u)-(G(u))_{B}\right\|_{p, B, w^{\alpha}}+\left\|\nabla\left(G(u)-(G(u))_{B}\right)\right\|_{p, B, w^{\alpha}} \\
& \leq \operatorname{diam}(B)^{-1} \cdot C_{2} \operatorname{diam}(B)\|d u\|_{p, \sigma_{1} B, w^{\alpha}}+C_{3}\|d u\|_{p, \sigma_{2} B, w^{\alpha}} \\
& \leq C_{4}\|d u\|_{p, \sigma B, w^{\alpha}},
\end{aligned}
$$


here $\sigma=\max \left(\sigma_{1}, \sigma_{2}\right)$ with $\sigma B \subset M$. Applying Lemma 1.5 and (2.20), we conclude that

$$
\left\|G(u)-(G(u))_{B}\right\|_{W^{1, p}(B), w^{\alpha}}^{p} \leq C_{5}\|d u\|_{p, \sigma B, w^{\alpha}}^{p} \leq C_{5}\left\|d^{*} v\right\|_{q, \sigma B, w^{\alpha}}^{q} .
$$

Therefore, we have completed the proof of Theorem 2.2.

\section{References}

[1] R. P. Agarwal and S. Ding, Advances in differential forms and the A-harmonic equation, Math. Comput. Modelling 37 (2003), no. 12-13, 1393-1426.

[2] S. Ding, Integral estimates for the Laplace-Beltrami and Green's operators applied to differential forms on manifolds, Z. Anal. Anwendungen 22 (2003), no. 4, 939-957.

[3] - The weak reverse Hölder inequality for conjugate A-harmonic tensors, preprint, 2004.

[4] F. W. Warner, Foundations of Differentiable Manifolds and Lie Groups, Graduate Texts in Mathematics, vol. 94, Springer-Verlag, New York, 1983.

[5] Y. Xing, Weighted integral inequalities for solutions of the A-harmonic equation, J. Math. Anal. Appl. 279 (2003), no. 1, 350-363.

[6]_, Analogues of the Poincaré inequality for conjugate A-harmonic tensor, preprint, 2004.

Yuming Xing: Department of Mathematics, Harbin Institute of Technology, Harbin, China

E-mail address: xyuming@hit.edu.cn 